\title{
Simultaneous Hot Electron and Hole Injection upon Excitation of Gold Surface Plasmon
}

Hattori, Yocefu; Abdellah, Mohamed; Meng, Jie; Zheng, Kaibo; Sá, Jacinto

Published in:

Journal of Physical Chemistry Letters

Link to article, DOI:

10.1021/acs.jpclett.9b01085

Publication date:

2019

Document Version

Publisher's PDF, also known as Version of record

Link back to DTU Orbit

Citation (APA):

Hattori, Y., Abdellah, M., Meng, J., Zheng, K., \& Sá, J. (2019). Simultaneous Hot Electron and Hole Injection upon Excitation of Gold Surface Plasmon. Journal of Physical Chemistry Letters, 10(11), 3140-3146. https://doi.org/10.1021/acs.jpclett.9b01085

\section{General rights}

Copyright and moral rights for the publications made accessible in the public portal are retained by the authors and/or other copyright owners and it is a condition of accessing publications that users recognise and abide by the legal requirements associated with these rights.

- Users may download and print one copy of any publication from the public portal for the purpose of private study or research.

- You may not further distribute the material or use it for any profit-making activity or commercial gain

- You may freely distribute the URL identifying the publication in the public portal 


\title{
Simultaneous Hot Electron and Hole Injection upon Excitation of Gold Surface Plasmon
}

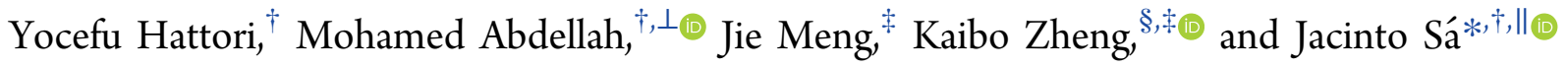 \\ ${ }^{\dagger}$ Physical Chemistry Division, Department of Chemistry, Ångström Laboratory, Uppsala University, 75120 Uppsala, Sweden \\ ${ }^{\ddagger}$ Department of Chemistry, Technical University of Denmark, DK-2800 Kongens Lyngby, Denmark \\ ${ }^{\S}$ Chemical Physics and NanoLund, Lund University, Box 124, 22100 Lund, Sweden \\ "Institute of Physical Chemistry, Polish Academy of Sciences, 01-224 Warsaw, Poland \\ ${ }^{\perp}$ Department of Chemistry, Qena Faculty of Science, South Valley University, 83523 Qena, Egypt
}

\section{Supporting Information}

ABSTRACT: We have successfully investigated the simultaneous injection of hot electrons and holes upon excitation of gold localized surface plasmon resonance (LSPR). The studies were performed on all-solid-state plasmonic system composed of titanium dioxide $\left(\mathrm{TiO}_{2}\right) /$ poly $(3,4$-ethylenedioxythiophene):poly(styrenesulfonic acid) (PEDOT:PSS) $\mathrm{p}-\mathrm{n}$ junctions with gold nanoparticles (Au NPs). The study revealed that both charge carriers are transferred within $200 \mathrm{fs}$ to the respective charge acceptors, exhibiting a free carrier transport behavior. We also confirmed that the transfer of charge carriers are accompanied by change in the initial relaxation dynamics of Au NPs.

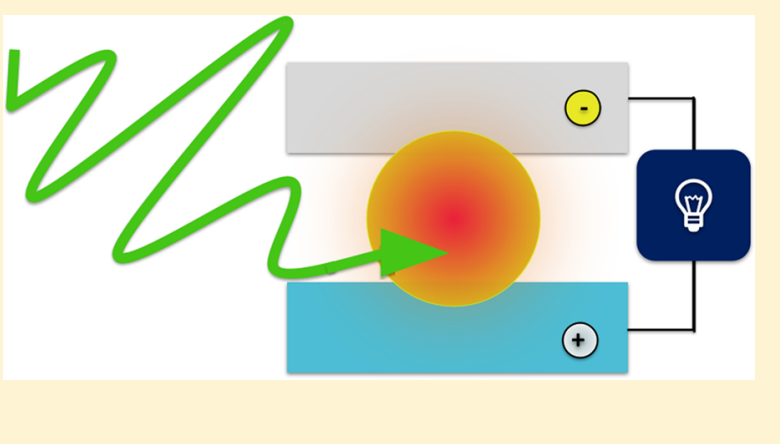

$\mathrm{T}$ he efficient conversion of light energy into electricity is key for sustainable human development. Photovoltaics have the potential to make a significant contribution to satisfy the energy requirements; however, issues related to efficiency, sustainability, and cost must be addressed with increased urgency. The main limitation of current technologies relates to the low absorbance of near-bandgap light. Metal nanoparticles (NPs) are strong light scatterers at wavelengths near the plasmon resonance, which is due to a collective oscillation of the conduction electrons in the metal. Hot carriers in plasmonic NPs can be generated both by direct excitation and by LSPR excitation and decay, ${ }^{1}$ which was confirmed spectroscopically in 2013 by Sá and co-workers. ${ }^{2}$ They demonstrated that excitation of Au LSPR resulted in the formation of hot carriers (electrons and holes) with the help of high-resolution X-ray absorption spectroscopy at the $\mathrm{Au} \mathrm{L}_{3}$ edge. Those hot electrons could be readily injected into a $\mathrm{TiO}_{2}$ conduction band (CB), confirming their usability for light conversion to electricity. Recently, it was demonstrated that a hot electrons counterpart (i.e., hot holes) could also be injected into p-type semiconductors with suitable valence band energy edges alignment upon LSPR excitation, ${ }^{3}$ corroborating previously reported photocatalysis studies ${ }^{4-6}$ and stimulating research into direct solar cells with LSPR nanostructures. ${ }^{7-10}$ LSPR excitation creates a non-Fermi distribution of electrons that rapidly relaxes to a Fermi distribution via electronelectron $(e-e)$ scattering, with relaxation dynamics dependent on induced transition (intra- or interband transition). The ultrafast nature of $\mathrm{e}-\mathrm{e}$ scattering processes in these materials ${ }^{11-13}$ demands transference of the plasmon-induced charger carriers to suitable transport layers for exploitation as photovoltaics. The energy of the electron-hole pairs is determined by the incoming photon energy; however, the carriers' energy distributions depend on LSPR decay processes. ${ }^{14}$ Herein is reported the ultrafast dynamics of hot electrons and holes formed via excitation of gold LSPR. The studies were performed on an all-solid-state plasmonic system composed of titanium dioxide $\left(\mathrm{TiO}_{2}\right) / \operatorname{poly}(3,4-$ ethylenedioxythiophene):poly(styrenesulfonic acid) (PEDOT:PSS) $\mathrm{p}-\mathrm{n}$ junction with gold nanoparticles (Au NPs). The study revealed that both charge carriers are transferred within 200 fs to the respective charge acceptors, exhibiting a free carrier transport behavior. A simplified scheme of the charge dynamics is depicted in Figure 1e.

The all-solid-state plasmonic system consists of a compact bottom layer of $\mathrm{TiO}_{2}$ NPs decorated with Au NPs under a top thin film layer of PEDOT:PSS. PEDOT is a semiconductor, which is degenerately doped by PSS to achieve a p-type conductive mechanism. ${ }^{15}$ Atomic force microscopy (AFM) measurements (Figure S2 in the Supporting Information) revealed a 200 and $40 \mathrm{~nm}$ layer thickness for the semiconductor and conducting polymer, respectively. The evaporation of a $2 \mathrm{~nm}$ of Au followed by annealing at $450{ }^{\circ} \mathrm{C}$ for 30 min in Ar flow leads to formation of particles with mean

Received: April 16, 2019

Accepted: May 22, 2019

Published: May 22, 2019 

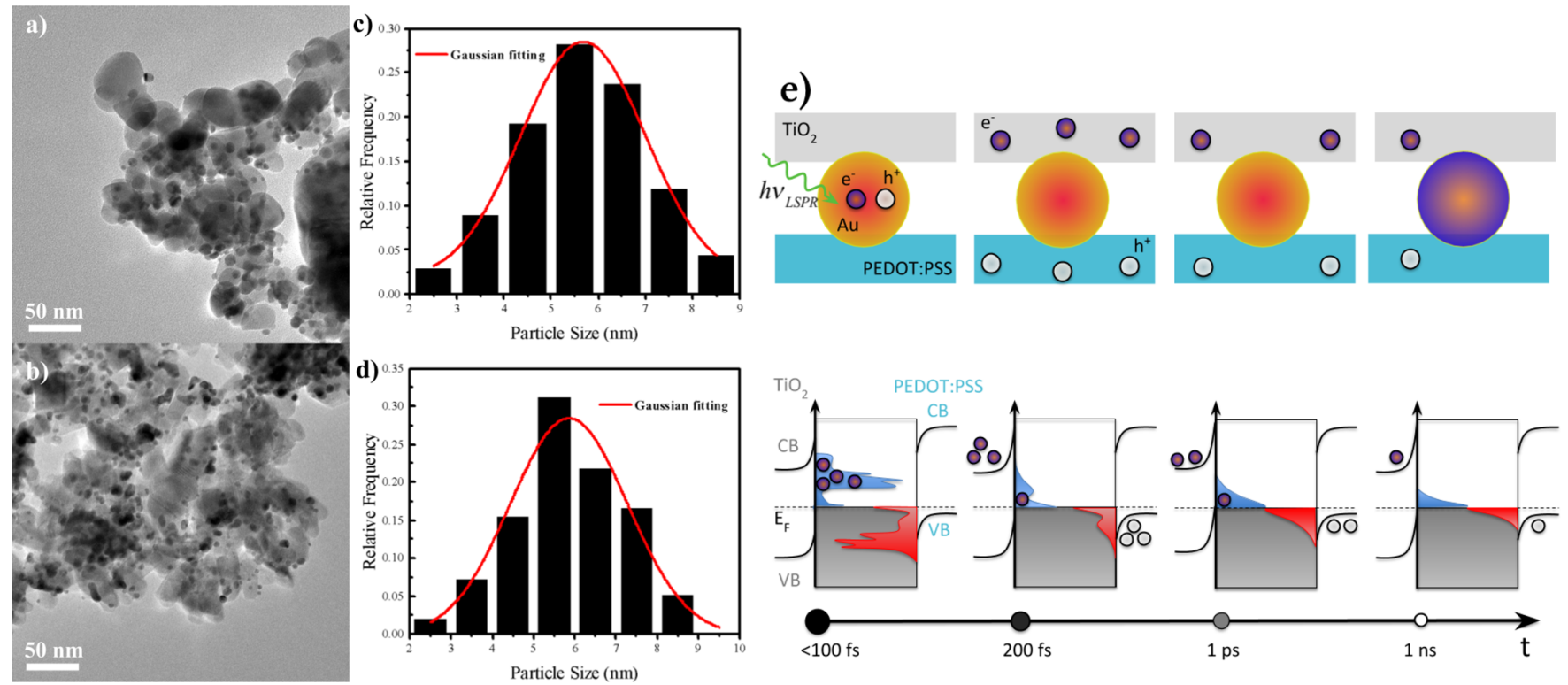

Figure 1. TEM images of Au NPs on $\mathrm{ZrO}_{2}$ (a) and $\mathrm{TiO}_{2}$ (b) and their respective particles size distributions (c), (d). Schematic picture of the dynamics of electrons and holes injected to $\mathrm{TiO}_{2}$ and PEDOT:PSS upon light excitation at $530 \mathrm{~nm}(\mathrm{e})$.

a)

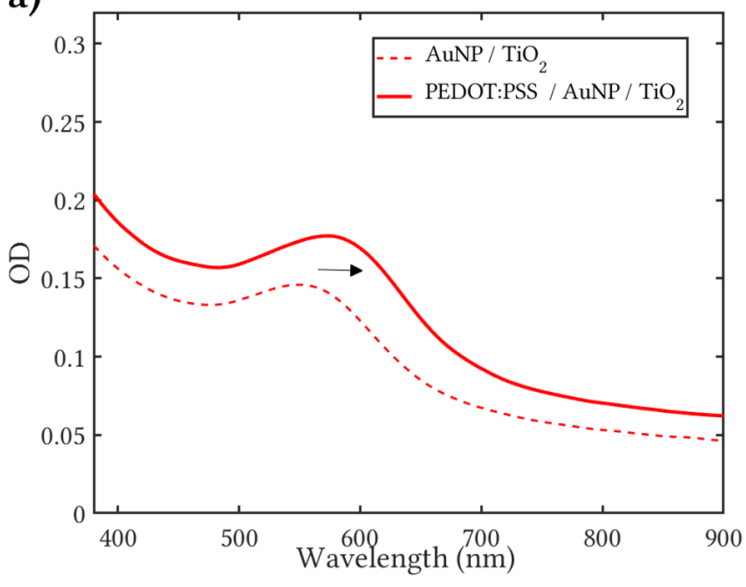

c)

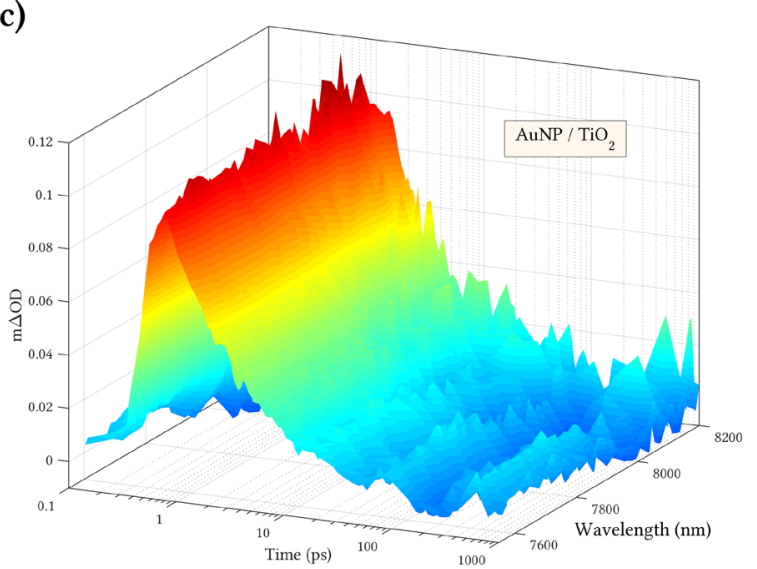

b)

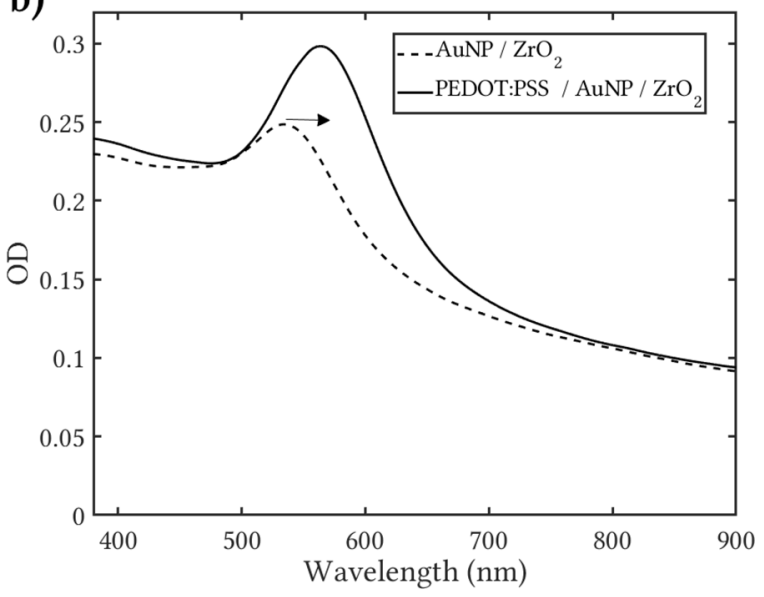

d)

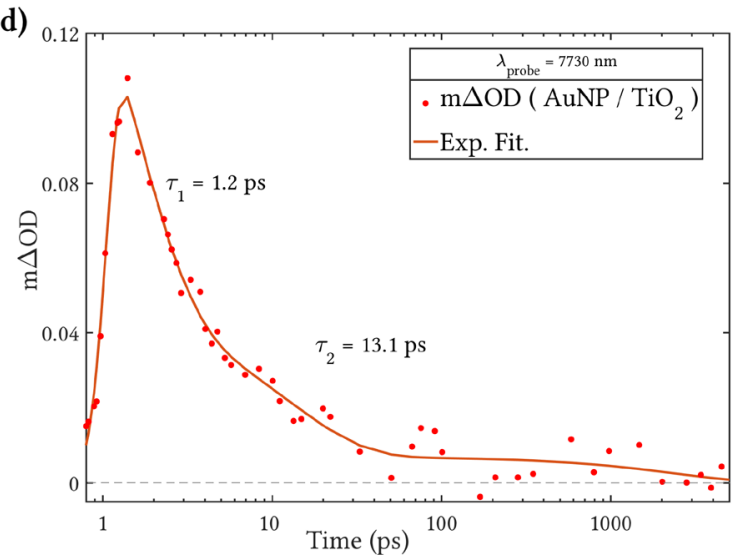

Figure 2. Absorbance spectrum of $\mathrm{AuNP}_{\mathrm{TiO}}$ (a) and $\mathrm{AuNP} / \mathrm{TiO}_{2}$ (b) with and without PEDOT:PSS. Transient Spectra of AuNP/TiO $(\mathrm{c})$ and kinetics extracted at $7730 \mathrm{~nm}(\mathrm{~d})$.

diameters of ca. $6 \mathrm{~nm}$, as is shown from transmission electron microscopy (TEM) measurements (Figure la-d). Further details of sample preparation are described in the Supporting Information.
The plasmon resonance is known to be sensitive to asymmetric dielectric perturbations. ${ }^{16}$ The surrounding dielectric mediums of Au NPs are changed anisotropically with the presence of PEDOT:PSS, thus distorting the plasmonic 

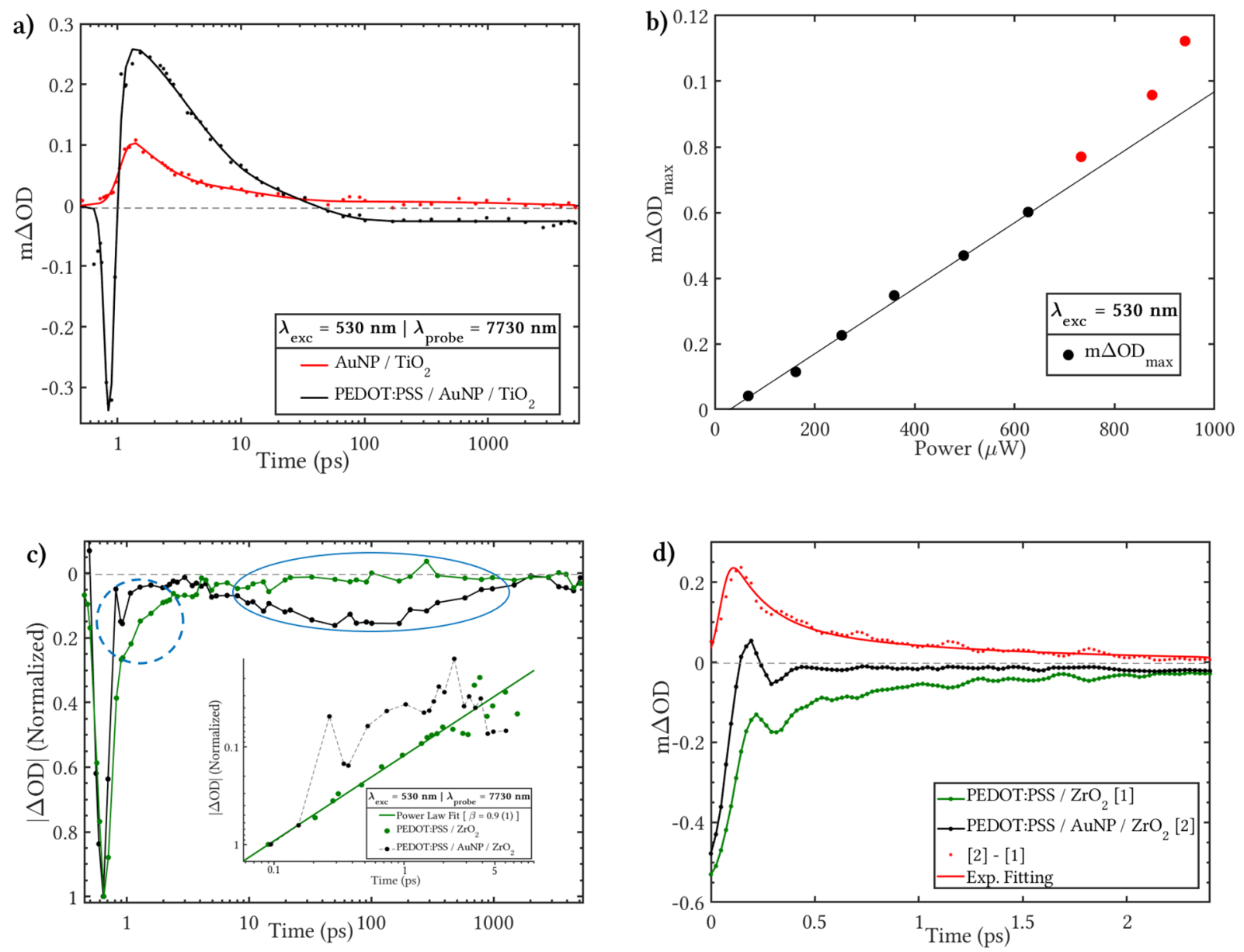

Figure 3. Kinetics at $7730 \mathrm{~nm}$ extracted from $\mathrm{AuNP}_{\mathrm{TiO}}$ (red) and PEDOT:PSS/AuNP/TiO $\left(\right.$ black) (a). $\mathrm{m} \Delta \mathrm{OD}_{\max }$ for different laser powers with fixed wavelength at $530 \mathrm{~nm}$ (b). Kinetics at $7730 \mathrm{~nm}$ of PEDOT:PSS $/ \mathrm{ZrO}_{2}$ with (black) and without (green) Au NPs. The inset shows the kinetics in $\log -\log$ presentation. Power-law decay fitting yielded $\beta=0.9(1)$ (c). Kinetics of holes in the conducting polymer extracted from the subtraction of PEDOT:PSS/AuNP/ $\mathrm{ZrO}_{2}$ by PEDOT:PSS/TiO 2 transient signals (d).

resonance, which leads to different shifts in the absorbance and scattering spectrum. This can be clearly observed by comparing the absorbance of the system when PEDOT:PSS is spin-coated (Figure 2a,b). The same argument can be used to explain the change in the spectra depending on which metal oxide $\left(\mathrm{TiO}_{2}, \mathrm{ZrO}_{2}\right)$ the Au NPs are attached to. The thin film of PEDOT:PSS have an extremely low absorption in the visible range; however, Raman measurements (Figure S3) reveal the typical features of the conducting polymer.

Free carriers (electrons and/or holes) have a strong absorption in the infrared (IR) spectra by exhibiting a broad featureless signal that increases in the entire mid-IR region. ${ }^{17}$ In transient infrared absorption spectroscopy (TIRAS), the temporal evolution of the difference in absorption $(\Delta \mathrm{OD}=$ $\left.\mathrm{OD}_{\text {pump }}-\mathrm{OD}_{\text {unpump }}\right)$ is measured and the positive signal is assigned to free carriers in the accepting materials $\left(\mathrm{TiO}_{2}\right.$ and/ or PEDOT:PSS), without interference from the Au LSPR process (see Figure S4). The kinetic traces were fitted using a sum of convoluted exponential functions (see eq S1) in order to estimate the rise time (charge carrier injection time) and decay time (charge carrier lifetime) of the signal. We investigated the systems using TIRAS, where the probe interval was $\lambda_{\text {probe }}=7600-8200 \mathrm{~nm}$ and the excitation wavelength was set to $\lambda_{\mathrm{exc}}=530 \mathrm{~nm}$ (near the Au LSPR peak). Initially, the system composed of only $\mathrm{Au} \mathrm{NPs}$ on $\mathrm{TiO}_{2}$ was measured. The time zero, i.e., the overlap in time of the pump and probe pulse, was shifted for plotting convenience. Figure $2 \mathrm{c}, \mathrm{d}$ shows the temporal evolution of the difference in absorption where the positive $\Delta \mathrm{OD}$ is ascribed to free carriers, in this case electrons in the $\mathrm{CB}$ of $\mathrm{TiO}_{2}$ injected from $\mathrm{Au}$ NPs upon optical excitation. The time associated with charge injection was estimated to be $\tau_{\text {rise }}=192 \mathrm{fs}$ by fitting the rising edge region.

The presence of conducting polymer thin film on AuNP/ $\mathrm{TiO}_{2}$ causes a dramatic change on the transient IR signal as it is compared in the Figure 3a. Three aspects can be promptly noted: the presence of PEDOT:PSS causes a bleach with a rapid recovery in the subpicosecond time regime following excitation, a negative signal at longer time delays, and a rise in the maximum positive amplitude by a factor of ca. 2.5 . The latter underlines the increase in the number of free carriers in the system. The time evolution of the spectrum comparing both systems can be viewed in Figure S4. Figure S5 shows that no positive transient signal is observed when optically exciting the PEDOT:PSS/ $\mathrm{TiO}_{2}$ system, therefore excluding any possibilities of the conducting polymer injecting electrons to the $\mathrm{TiO}_{2}$ semiconductor. The dependence of the laser power with the maximum positive $\triangle O D$ was studied for PEDOT:PSS/AuNP/ $/ \mathrm{TiO}_{2}$ system and a deviation from the linear range occurs for values higher than $620 \mu \mathrm{W}$ (Figure 3b). In our experiments the excitation laser power was kept to ca. $560 \mu \mathrm{W}$ during all the measurements, i.e., in the linear region. 
a)
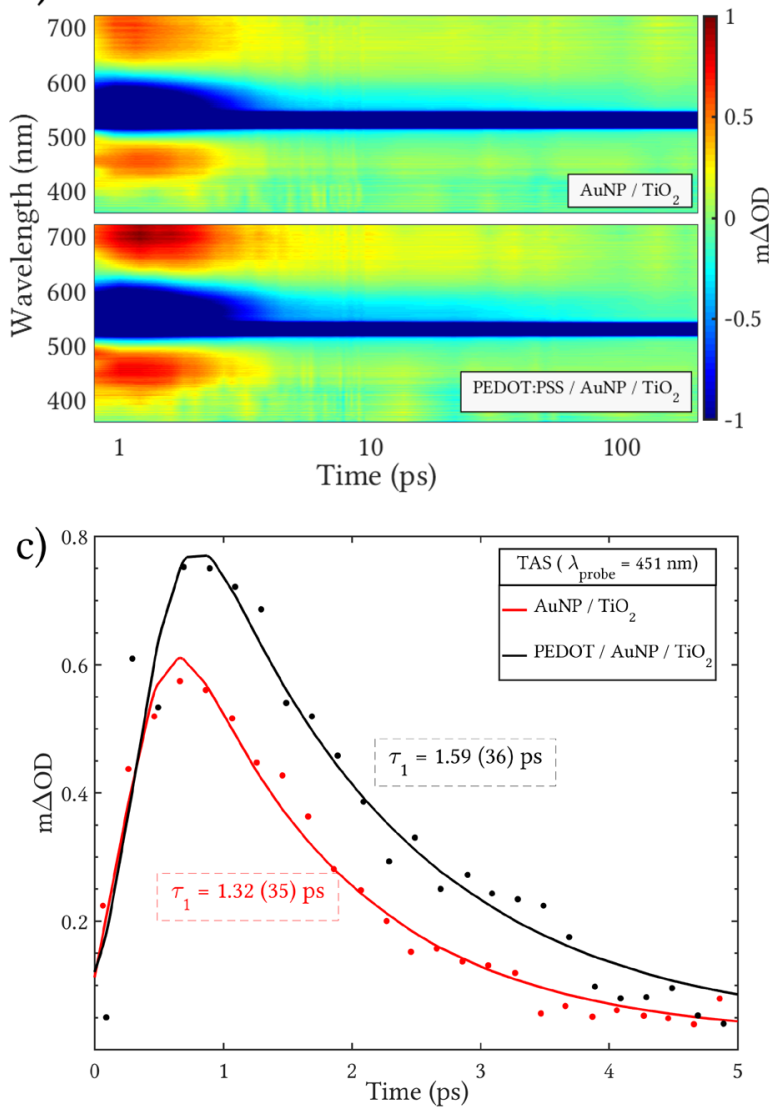

b)
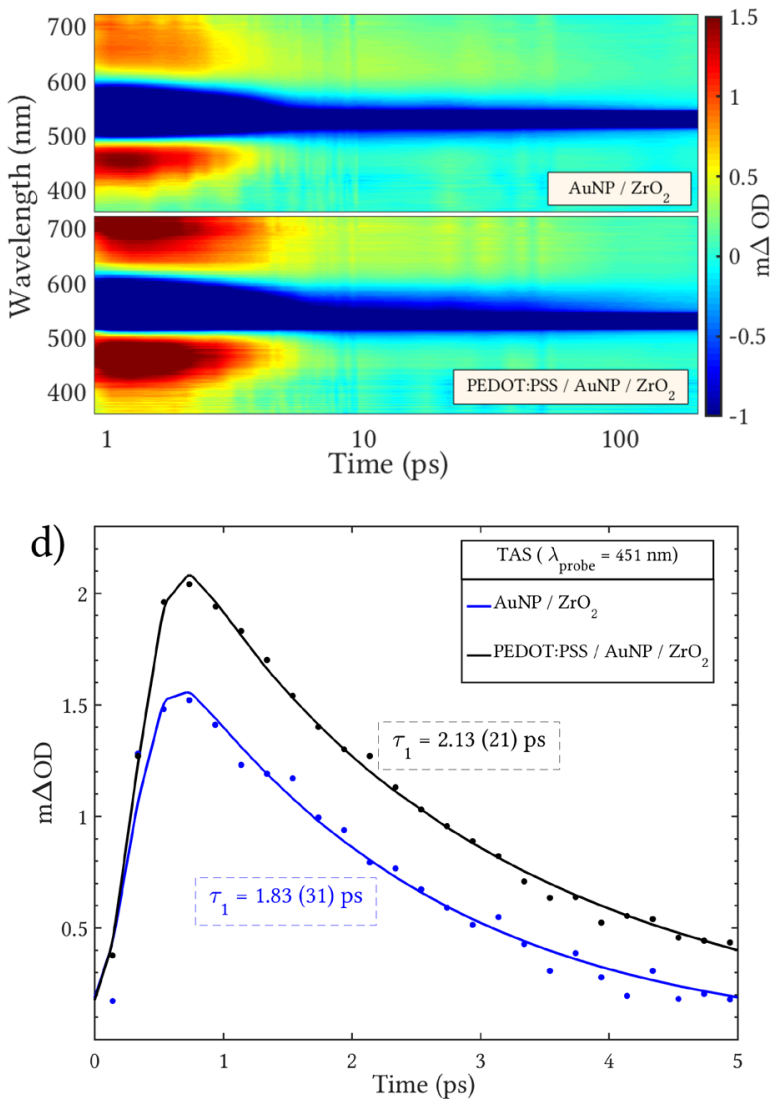

Figure 4. (a) Temporal evolution of the spectrum probed in the visible region of PEDOT:PSS/Au NPs/TiO 2 and (b) PEDOT:PSS/Au NPs/ $\mathrm{ZrO}_{2}$. Temporal evolution of (c) AuNP/TiO ${ }_{2}$ and (d) AuNP/ZrO $\mathrm{Zr}_{2}$ with and without PEDOT:PSS probed at $451 \mathrm{~nm}$. The dashed rectangles contain the $\mathrm{e}-\mathrm{ph}$ coupling times obtained by an exponential function.

Such unusual upward divergence from the linearity is characteristic for plasmonic materials. ${ }^{18}$ Nevertheless, studies of this nonlinear mechanism is beyond the scope of this work.

In order to investigate the aforementioned features, the same measurements were performed with a sample containing $\mathrm{ZrO}_{2}$ instead of $\mathrm{TiO}_{2}$, therefore avoiding electron injection from $\mathrm{Au}$ NPs and restricting its interaction triggered by light only with PEDOT:PSS. TIRAS measurement of PEDOT:PSS spincoated over an insulating layer of $\mathrm{ZrO}_{2}$ exhibits a bleach that has been attributed to the electronic thermalization of the excited charge carriers in the polymeric conductor. ${ }^{19}$ Our thin film conducting polymer has an extremely low absorption in the visible region, but an intense broad band absorption in the IR (Figure S7). The presence of free charge carriers in the doped PEDOT are responsible for this band and has been interpreted in terms of the localization-modified Drude model (LMD) with good quantitative agreement with the reflectance spectrum. ${ }^{20}$ The LMD model, which is a first-order correction of the Drude model in the regime of weak localization, has been extensively used to describe metal-insulator transitions in conducting polymer. ${ }^{21,22}$ This implies that the IR absorption is due to motion of charge carriers (intraband transition) in the electric field of the light. Meskers et al. ${ }^{23}$ observed similar frequency dependence between opto-electrical measurements and photoinduced absorption at IR probe energies, suggesting that the optical and electrical signal are due to sample heating. Therefore, it can be rationalized that with the increase in the electronic temperature induced by the laser pulse, the number of states available to the carriers will be modified, thus leading to a change in the absorption spectra.

By comparing both curves in Figure 3c, it is possible to first observe a faster decay (dashed blue circle) when the plasmonic NPs are present. Since thermal dissipation from the metal to the surrounding medium does not happen in the subpicosecond time scale, ${ }^{24}$ this effect can be disregarded within this time region but is directly related to the second one (blue elipse, Figure 3c) and will be discussed later. Previous studies of fully undoped PEDOT indicates a band gap $>1.5 \mathrm{eV}^{25,26}$ Notwithstanding, irradiation of PEDOT:PSS $/ \mathrm{ZrO}_{2}$ with $\lambda_{\text {exc }}=$ $530 \mathrm{~nm}(2.34 \mathrm{eV})$ leads to the same power law decay behavior $\left(1 / t^{\beta}\right)$ with $\beta=0.9 \pm 0.1$ observed by Meskers et al. $\left(\beta_{\text {lit }}=0.8\right.$ $\pm 0.1)$ when pump/probed at $1440 \mathrm{~nm}(0.86 \mathrm{eV}) .{ }^{19}$ Therefore, we concluded that photoconduction from photogenerated charge in our thin film of PEDOT:PSS is unlikely. Moreover, a deviation from the power law decay behavior in the initial transient decay can only be observed when Au NPs is present (insert of Figure 3c); Figure S6 compares the kinetics of this system with $\lambda_{\mathrm{exc}}=800 \mathrm{~nm}$, i.e., far from the LSPR peak and a similar slower decay to PEDOT:PSS $/ \mathrm{ZrO}_{2}$ is observed. This leads us to conclude that the divergence from power decay law behavior is caused by hole injection upon $\mathrm{Au}$ LSPR excitation and not from PEDOT:PSS optical excitation. In addition, the negative signal after 0.5 ps is significantly more intense for the sample without $\mathrm{Au}$, suggesting that a positive signal offsets the trace consistent with holes populating PEDOT:PSS valence band. Figure S4 indicates that no IR 
Table 1. e-ph Coupling Time $(\tau)$ and $\left.\operatorname{lm} \Delta O D\right|_{\max }$ of Different Systems and Probing Wavelengths

\begin{tabular}{|c|c|c|c|c|c|c|}
\hline & \multicolumn{2}{|c|}{$451 \mathrm{~nm}$} & \multicolumn{2}{|c|}{$575 \mathrm{~nm}$} & \multicolumn{2}{|c|}{$700 \mathrm{~nm}$} \\
\hline & $\left.\operatorname{lm} \Delta O D\right|_{\max }$ & $\tau(\mathrm{ps})$ & $\left.\operatorname{lm} \Delta O D\right|_{\max }$ & $\tau(\mathrm{ps})$ & $\left.\operatorname{lm} \Delta \mathrm{OD}\right|_{\max }$ & $\tau(\mathrm{ps})$ \\
\hline $\mathrm{AuNP} / \mathrm{TiO}_{2}$ & 0.9 & $1.32 \pm 0.35$ & 1.8 & $1.30 \pm 0.07$ & 0.63 & $1.37 \pm 0.40$ \\
\hline \multirow{3}{*}{ PEDOT:PSS/AuNP/ $/ \mathrm{TiO}_{2}$} & 1.1 & $1.59 \pm 0.36$ & 2.2 & $1.42 \pm 0.11$ & 1.1 & $1.43 \pm 0.52$ \\
\hline & \multicolumn{2}{|c|}{$451 \mathrm{~nm}$} & \multicolumn{2}{|c|}{$560 \mathrm{~nm}$} & \multicolumn{2}{|c|}{$700 \mathrm{~nm}$} \\
\hline & $|\mathrm{m} \Delta \mathrm{OD}|_{\max }$ & $\tau(\mathrm{ps})$ & $|\mathrm{m} \Delta \mathrm{OD}|_{\max }$ & $\tau(\mathrm{ps})$ & $|\mathrm{m} \Delta \mathrm{OD}|_{\max }$ & $\tau(\mathrm{ps})$ \\
\hline $\mathrm{AuNP} / \mathrm{ZrO}_{2}$ & 2 & $1.83 \pm 0.31$ & 4.6 & $1.69 \pm 0.09$ & 1.0 & $1.49 \pm 0.33$ \\
\hline PEDOT:PSS/AuNP/ZrO ${ }_{2}$ & 2.5 & $2.13 \pm 0.21$ & 5.4 & $1.84 \pm 0.12$ & 1.9 & $2.22 \pm 0.24$ \\
\hline
\end{tabular}

signal is observed from $\mathrm{AuNP} / \mathrm{ZrO}_{2}$. Figure $3 \mathrm{~d}$ depicts the same kinetics at $7730 \mathrm{~nm}$ but with more dense delay points. The data from each system were interpolated and subtracted to obtain the transient signal arising from holes in PEDOT:PSS. In the presence of $\mathrm{Au} \mathrm{NPs,} \mathrm{there} \mathrm{is} \mathrm{a} \mathrm{clear} \mathrm{positive} \mathrm{signal}$ associated with hole transfer to PEDOT:PSS, which is not present in the sample without Au NPs (see figure S4 for the transient spectra). The fitting of the rising edge resulted in a value significantly shorter than our timing resolution, thus we can only state that hole injection occurs within our instrument response function. The negative signal contribution (ca. $t>3$ ps, Figure 3c) resulting from the thermal dissipation from $\mathrm{Au}$ NPs to PEDOT:PSS prevents an accurate analysis of hole kinetics for longer time delays.

Figure $4 \mathrm{a}, \mathrm{b}$ shows the time evolution of $\Delta \mathrm{OD}$ for samples with and without PEDOT:PSS upon excitation at also $530 \mathrm{~nm}$ but probing in the visible region. The $2 \mathrm{D}$ graph depicts the typical transient features of Au NPs with two positive "winglets" and a bleach region measured by ultrafast transient absorption (TAS), which is mainly governed by electronphonon $(\mathrm{e}-\mathrm{ph})$ scattering and have been extensively studied and modeled elsewhere. ${ }^{27,28}$ The increase in the electronic temperature, followed by its thermalization to the lattice, induced by the pump laser modifies the dielectric constant of the particles causing transient changes in the spectrum. The transient behavior of Au NPs has been modeled and excellently reproduces the experimental curves by taking into account the temporal change in the Drude-like $\epsilon_{\text {intra }}$ and interband $\epsilon_{\text {inter }}$ terms on the dielectric function. ${ }^{29}$ Several transient absorption experiments have been performed to determine this coupling constant, but these measurements are problematic since the relaxation time depends on pump laser intensity. ${ }^{30,31}$ In order to overcome this, experiments in the literature performed measurements with very low excitation powers so the electron distribution is only slighty modified and the results were extrapolated to zero power. ${ }^{28,32}$ In general, the e-ph coupling time obtained is ca. 1 ps for high conductivity metals like $\mathrm{Au}$, $\mathrm{Ag}$, and $\mathrm{Cu}$. Furthermore, experimental studies confirmed the absence of size-dependence on the initial thermalization of $\mathrm{Au}$ NPs. ${ }^{33}$

The kinetics extracted at $451 \mathrm{~nm}$ for the system on $\mathrm{TiO}_{2}$ and $\mathrm{ZrO}_{2}$ are shown in Figure 4c,d. A longer lifetime for the first component ( $\mathrm{e}-\mathrm{ph}$ coupling) can be obtained when PEDOT:PSS is present, from $\tau=1.32 \pm 0.35 \rightarrow 1.59 \pm$ 0.36 ps for the system with $\mathrm{TiO}_{2}$ and $\tau=1.83 \pm 0.31 \rightarrow 2.17 \pm$ $0.21 \mathrm{ps}$ for the one with $\mathrm{ZrO}_{2}$. The Table 1 list the $\tau$ values of the system with and without PEDOT:PSS obtained in three different regions: the bleach's bottom and the two positive peaks region. Although the $\tau$ differences obtained are within experimental uncertainty, the mean $\mathrm{e}-\mathrm{ph}$ coupling time values are slightly increased in all regions. Since the coupling period depends on the initial electronic temperature, ${ }^{34}$ the prolongation in time corroborates with holes being injected into PEDOT:PSS from Au NPs. The opposite effect, i.e., the decrease in the $\mathrm{e}-\mathrm{ph}$ coupling time, is observed when the system contains $\mathrm{TiO}_{2}$ (electron acceptor) instead of $\mathrm{ZrO}_{2}$.

Following the internal e-e scattering and $\mathrm{e}-\mathrm{ph}$ coupling process, the hot nanoparticle equilibrates with the environment. Heat diffusion within the particle is very fast for metals and is often neglected. ${ }^{35}$ The description of the sequence of events, namely, $\mathrm{e}-\mathrm{e}$ scattering, $\mathrm{e}-\mathrm{ph}$ coupling, and heat dissipation after optical excitation, is commonly divided into different time scales. However, they are not accurate since they depend of different parameters like size, shape, dielectric medium, etc., although it offers a good starting point for discussion. Hartland et al. ${ }^{36}$ have studied the energy dissipation of $\mathrm{Au}$ NPs to their surroundings with pump-probe spectroscopy and verified that, for very small particles (ca. $4 \mathrm{~nm}$ ), significant energy loss occurs before electron and phonons reach thermal equilibrium. Taking this into account, we suggest that the component related to the heat dissipation into the surroundings (highlight in Figure $5, \lambda_{\text {probe }}=700 \mathrm{~nm}$ ) is the cause of the negative absorption on the transient dynamics of PEDOT:PSS beginning around 3 ps.

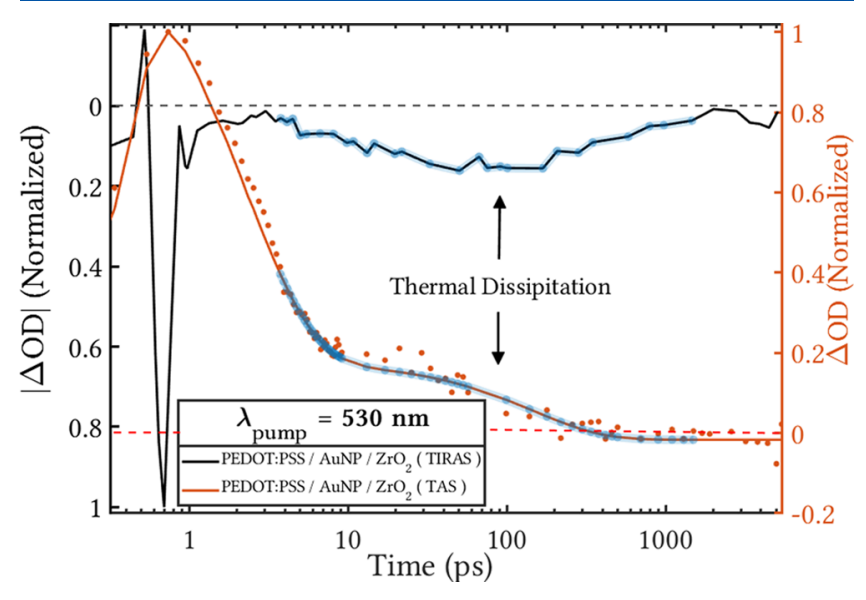

Figure 5. TIRAS and TAS kinetics of PEDOT:PSS/AuNP/ $/ \mathrm{ZO}_{2}$ probed at 7730 and $700 \mathrm{~nm}$, respectively.

In conclusion, we showed that electronic thermalization of PEDOT:PSS is altered due to hole injection from Au NPs to the conducting polymer, which resulted in an increase of ca. 2.5 of the signal related to free carriers in the system. One could argue that initially only electrons are injected to $\mathrm{CB}$ of $\mathrm{TiO}_{2}$, leading to a successive Fermi level shift to a point where finally holes could be transferred to PEDOT:PSS. Nevertheless, our results showed that electron and hole injection occur within 200 fs. The result is consistent with the work of 
Furube et al. where the electron injection time from Au NPs into $\mathrm{TiO}_{2}$ was estimated to happen within $240 \mathrm{fs}^{37}$ The increase in the $\mathrm{e}-\mathrm{ph}$ coupling time with the presence of PEDOT:PSS and decrease when $\mathrm{TiO}_{2}$ is used instead of $\mathrm{ZrO}_{2}$ reflects the influence of charge injection from plasmonic nanoparticles on its initial relaxation dynamics. Although charge recombination from PEDOT:PSS to $\mathrm{TiO}_{2}$ is likely to be playing a role, this work suggests that with further improvements, such as insertion of a thin insulating layer between the conducting polymer and the semiconductor, this system has the potential applicability of an all-solid-state plasmonic photovoltaic device with light absorption in the visible region.

\section{ASSOCIATED CONTENT}

\section{S Supporting Information}

The Supporting Information is available free of charge on the ACS Publications website at DOI: 10.1021/acs.jpclett.9b01085.

Experimental details including samples preparation and characterization techniques, and some supporting data (PDF)

\section{AUTHOR INFORMATION}

\section{Corresponding Author}

*E-mail: jacinto.sa@kemi.uu.se

\section{ORCID}

Mohamed Abdellah: 0000-0002-6875-5886

Kaibo Zheng: 0000-0002-7236-1070

Jacinto Sá: 0000-0003-2124-9510

\section{Notes}

The authors declare no competing financial interest.

\section{ACKNOWLEDGMENTS}

The authors thank Uppsala University and the Swedish Research Council for financial support. J.M. acknowledge financial support from China Scholarship Council.

\section{REFERENCES}

(1) Manjavacas, A.; Liu, J. G.; Kulkarni, V.; Nordlander, P. PlasmonInduced Hot Carriers in Metallic Nanoparticles. ACS Nano 2014, 8, $7630-7638$

(2) Sá, J.; Tagliabue, G.; Friedli, P.; Szlachetko, J.; Rittmann-Frank, M. H.; Santomauro, F. G.; Milne, C. J.; Sigg, H. Direct observation of charge separation on Au localized surface plasmons. Energy Environ. Sci. 2013, 6, 3584-3588.

(3) Tagliabue, G.; DuChene, J. S.; Abdellah, M.; Habib, A.; Hattori, Y.; Zheng, K.; Canton, S. E.; Gosztola, D. J.; Cheng, W.-H.; Sundararaman, R.; Sa, J.; Atwater, H. A. Ultrafast Studies of Hot-Hole Dynamics in $\mathrm{Au} / \mathrm{p}-\mathrm{GaN}$ Heterostructures. arXiv e-prints 2018, https://arxiv.org/abs/1810.04238.

(4) Mubeen, S.; Lee, J.; Singh, N.; Krämer, S.; Stucky, G. D.; Moskovits, M. An autonomous photosynthetic device in which all charge carriers derive from surface plasmons. Nat. Nanotechnol. 2013, $8,247-251$.

(5) Yu, S.; Wilson, A. J.; Heo, J.; Jain, P. K. Plasmonic Control of Multi-Electron Transfer and C-C Coupling in Visible-Light-Driven $\mathrm{CO}_{2}$ Reduction on Au Nanoparticles. Nano Lett. 2018, 18, 21892194.

(6) DuChene, J. S.; Tagliabue, G.; Welch, A. J.; Cheng, W.-H.; Atwater, H. A. Hot Hole Collection and Photoelectrochemical $\mathrm{CO}_{2}$ Reduction with Plasmonic $\mathrm{Au} / \mathrm{p}-\mathrm{GaN}$ Photocathodes. Nano Lett. 2018, 18, 2545-2550.

(7) Atwater, H. A.; Polman, A. Plasmonics for improved photovoltaic devices. Nat. Mater. 2010, 9, 205-213.
(8) Hamann, T. W.; Jensen, R. A.; Martinson, A. B.; Van Ryswyk, H.; Hupp, J. T. Advancing beyond current generation dye-sensitized solar cells. Energy Environ. Sci. 2008, 1, 66-78.

(9) Nakamura, K.; Oshikiri, T.; Ueno, K.; Wang, Y.; Kamata, Y.; Kotake, Y.; Misawa, H. Properties of Plasmon-Induced Photoelectric Conversion on a $\mathrm{TiO}_{2} / \mathrm{NiO} \mathrm{p}-\mathrm{n}$ Junction with Au Nanoparticles. J. Phys. Chem. Lett. 2016, 7, 1004-1009.

(10) Reineck, P.; Lee, G. P.; Brick, D.; Karg, M.; Mulvaney, P.; Bach, U. A solid-state plasmonic solar cell via metal nanoparticle selfassembly. Adv. Mater. 2012, 24, 4750-4755.

(11) Juhasz, T.; Elsayed-Ali, H. E.; Smith, G. O.; Suárez, C.; Bron, W. E. Direct measurements of the transport of nonequilibrium electrons in gold films with different crystal structures. Phys. Rev. B: Condens. Matter Mater. Phys. 1993, 48, 15488-15491.

(12) Hodak, J. H.; Martini, I.; Hartland, G. V. Spectroscopy and Dynamics of Nanometer-Sized Noble Metal Particles. J. Phys. Chem. B 1998, 102, 6958-6967.

(13) Liu, J. G.; Zhang, H.; Link, S.; Nordlander, P. Relaxation of Plasmon-Induced Hot Carriers. ACS Photonics 2018, 5, 2584-2595.

(14) Zheng, B. Y.; Zhao, H.; Manjavacas, A.; McClain, M.; Nordlander, P.; Halas, N. J. Distinguishing between plasmon-induced and photoexcited carriers in a device geometry. Nat. Commun. 2015, 6, 7796 .

(15) Khodagholy, D.; Rivnay, J.; Sessolo, M.; Gurfinkel, M.; Leleux, P.; Jimison, L. H.; Stavrinidou, E.; Herve, T.; Sanaur, S.; Owens, R. M.; Malliaras, G. G. High transconductance organic electrochemical transistors. Nat. Commun. 2013, 4, 2133.

(16) Kelly, K. L.; Coronado, E.; Zhao, L. L.; Schatz, G. C. The Optical Properties of Metal Nanoparticles: The Influence of Size, Shape, and Dielectric Environment. J. Phys. Chem. B 2003, 107, 668677.

(17) Panayotov, D. A.; Burrows, S. P.; Morris, J. R. Infrared Spectroscopic Studies of Conduction Band and Trapped Electrons in UV-Photoexcited, H-Atom n-Doped, and Thermally Reduced $\mathrm{TiO}_{2}$. J. Phys. Chem. C 2012, 116, 4535-4544.

(18) Pavliuk, M. V.; Gutiérrez Álvarez, S.; Hattori, Y.; Messing, M. E.; Czapla-Masztafiak, J.; Szlachetko, J.; Silva, J. L.; Araujo, C. M.; A. Fernandes, D. L.; Lu, L.; Kiely, C. J.; Abdellah, M.; Nordlander, P.; Sá, J. Hydrated Electron Generation by Excitation of Copper Localized Surface Plasmon Resonance. J. Phys. Chem. Lett. 2019, 10, 17431749.

(19) Meskers, S.; Van Duren, J.; Janssen, R. Thermally Induced Transient Absorption of Light by Poly(3,4 ethylenedioxythiophene):Poly(styrene sulfonic acid) (PEDOT:PSS) Films: A way to probe charge-carrier thermalization process. $A d v$. Funct. Mater. 2003, 13, 805-810.

(20) Chang, Y.; Lee, K.; Kiebooms, R.; Aleshin, A.; Heeger, A. Reflectance of conducting poly(3,4-ethylenedioxythiophene). Synth. Met. 1999, 105, 203-206.

(21) Lee, K.; Heeger, A. J. Crossover to negative dielectric response in the low-frequency spectra of metallic polymers. Phys. Rev. B: Condens. Matter Mater. Phys. 2003, 68, 035201.

(22) Lee, K.; Menon, R.; Yoon, C. O.; Heeger, A. J. Reflectance of conducting polypyrrole: Observation of the metal-insulator transition driven by disorder. Phys. Rev. B: Condens. Matter Mater. Phys. 1995, 52, 4779-4787.

(23) Meskers, S. C. J.; van Duren, J. K. J.; Janssen, R. A. J. Stimulation of electrical conductivity in a $\pi$-conjugated polymeric conductor with infrared light. J. Appl. Phys. 2002, 92, 7041.

(24) Hartland, G. V. Optical Studies of Dynamics in Noble Metal Nanostructures. Chem. Rev. 2011, 111, 3858-3887.

(25) Apperloo, J. J.; Groenendaal, L. B.; Verheyen, H.; Jayakannan, M.; Janssen, R. A. J.; Dkhissi, A.; Beljonne, D.; Lazzaroni, R.; Brédas, J.-L. Optical and Redox Properties of a Series of 3,4-Ethylenedioxythiophene Oligomers. Chem. - Eur. J. 2002, 8, 2384-2396.

(26) Tran-Van, F.; Garreau, S.; Louarn, G.; Froyer, G.; Chevrot, C. Fully undoped and soluble oligo(3,4-ethylenedioxythiophene)s: Spectroscopic study and electrochemical characterization. J. Mater. Chem. 2001, 11, 1378-1382. 
(27) Hodak, J. H.; Martini, I.; Hartland, G. V. Spectroscopy and Dynamics of Nanometer-Sized Noble Metal Particles. J. Phys. Chem. B 1998, 102, 6958-6967.

(28) Voisin, C.; Del Fatti, N.; Christofilos, D.; Valle, F. Ultrafast Electron Dynamics and Optical Nonlinearities in Metal Nanoparticles. J. Phys. Chem. B 2001, 105, 2264-2280.

(29) Inouye, H.; Tanaka, K.; Tanahashi, I.; Hirao, K. Ultrafast dynamics of nonequilibrium electrons in a gold nanoparticle system. Phys. Rev. B: Condens. Matter Mater. Phys. 1998, 57, 11334-11340.

(30) Groeneveld, R. H. M.; Sprik, R.; Lagendijk, A. Femtosecond spectroscopy of electron-electron and electron-phonon energy relaxation in Ag and Au. Phys. Rev. B: Condens. Matter Mater. Phys. 1995, 51, 11433-11445.

(31) Feldstein, M. J.; Keating, C. D.; Liau, Y.-H.; Natan, M. J.; Scherer, N. F. Electronic Relaxation Dynamics in Coupled Metal Nanoparticles. J. Am. Chem. Soc. 1997, 119, 6638-6647.

(32) Arbouet, A.; Voisin, C.; Christofilos, D.; Langot, P.; Fatti, N. D.; Vallée, F.; Lermé, J.; Celep, G.; Cottancin, E.; Gaudry, M.; Pellarin, M.; Broyer, M.; Maillard, M.; Pileni, M. P.; Treguer, M. Electron-Phonon Scattering in Metal Clusters. Phys. Rev. Lett. 2003, 90, 177401.

(33) Minutella, E.; Schulz, F.; Lange, H. Excitation-Dependence of Plasmon-Induced Hot Electrons in Gold Nanoparticles. J. Phys. Chem. Lett. 2017, 8, 4925-4929.

(34) Hohlfeld, J.; Wellershoff, S.-S.; Güdde, J.; Conrad, U.; Jähnke, V.; Matthias, E. Electron and lattice dynamics following optical excitation of metals. Chem. Phys. 2000, 251, 237-258.

(35) Tas, G.; Maris, H. J. Electron diffusion in metals studied by picosecond ultrasonics. Phys. Rev. B: Condens. Matter Mater. Phys. 1994, 49, 15046-15054.

(36) Hu, M.; Hartland, G. V. Heat Dissipation for Au Particles in Aqueous Solution: Relaxation Time versus Size. J. Phys. Chem. B 2002, 106, 7029-7033.

(37) Furube, A.; Du, L.; Hara, K.; Katoh, R.; Tachiya, M. Ultrafast Plasmon-Induced Electron Transfer from Gold Nanodots into $\mathrm{TiO}_{2}$ Nanoparticles. J. Am. Chem. Soc. 2007, 129, 14852-14853. 